EPSC Abstracts

Vol. 14, EPSC2020-549, 2020

https://doi.org/10.5194/epsc2020-549

Europlanet Science Congress 2020

(C) Author(s) 2021. This work is distributed under

the Creative Commons Attribution 4.0 License.

\title{
Spectral properties of 2020 AV2, the first known asteroid orbiting inside Venus orbit
}

Marcel Popescu ${ }^{1}$, Julia de León ${ }^{2,3}$, Carlos de la Fuente Marcos ${ }^{4}$, Ovidiu Văduvescu ${ }^{2,5}$, Raúl de la Fuente Marcos $^{6}$, Javier Licandro ${ }^{2,3}$, Viktoria Pinter ${ }^{5,7}$, Eri Tatsumi ${ }^{2,3,8}$, and Lucian Curelaru ${ }^{9}$

${ }^{1}$ Astronomical Institute of the Romanian Academy, 5 Cut $\square$ itul de Argint, 040557 Bucharest, Romania (mpopescu@aira.astro.ro)

${ }^{2}$ Instituto de Astrofísica de Canarias (IAC), C/Vía Láctea s/n, 38205 La Laguna, Tenerife, Spain

${ }^{3}$ Departamento de Astrofísica, Universidad de La Laguna, 38206 La Laguna, Tenerife, Spain

${ }^{4}$ Universidad Complutense de Madrid, Ciudad Universitaria, E-28040 Madrid, Spain

${ }^{5}$ Isaac Newton Group of Telescopes (ING), Apto. 321, E-38700 Santa Cruz de la Palma, Canary Islands, Spain

${ }^{6}$ AEGORA Research Group, Facultad de CC. Matemáticas, Universidad Complutense de Madrid, Spain

${ }^{7}$ School of Doctoral Sciences, The University of Craiova, Str. A. I. Cuza nr. 13, 200585 Craiova, Romania

${ }^{8}$ Department of Earth and Planetary Science, The University of Tokyo, Bunkyo-ku, Tokyo, Japan

${ }^{9}$ Amateur Astronomer, Bras $\square$ ov, Romania

Recent numerical simulations (Greenstreet et al. 2012, Granvik et al. 2018) predicted the existence of a population of small bodies that is orbiting entirely inside Venus orbit. They could represent about $0.22 \%$ of the steady-state near-Earth asteroids (NEAs). These asteroids are called Vatiras (by analog with the Atira-class NEAs) or Interior to Venus Orbit Objects. However, only at the beginning of this year (January 4, 2020) the first one was discovered by Zwicky Transient Facility (Bolin et al. 2020). It is called $2020 \mathrm{AV}_{2}$ and has the aphelion at $0.654 \mathrm{AU}$, and the perihelion at $0.457 \mathrm{AU}$.

The dynamical history of this object has been explored using $N$-body simulations (de la Fuente Marcos \& de la Fuente Marcos 2020). It has been shown that $2020 \mathrm{AV}_{2}$ was a former Atira-class, and perhaps a former Aten-class asteroid, which reached the Vatira orbit relatively recently in astronomical terms, $\square 10^{5} \mathrm{yr}$ (within $9 \sigma$ confidence level). Similar results have also been reported by Greenstreet (2020).

The orbit of $2020 \mathrm{AV}_{2}$ makes it a peculiar case compared with those of the near-Earth asteroids. It is subjected to high temperature, strong solar wind irradiation, and close approaches to Mercury and (more distant) Venus. In this context, we carried out an observing run aimed at obtaining spectroscopic and photometric data for 2020 AV2. We used the $2.56 \mathrm{~m}$ Nordic Optical Telescope (NOT) and 4.2m William Herschel Telescope (WHT), both located at El Roque de los Muchachos Observatory in La Palma, Canary Islands (Spain). The observations were performed on the evenings of January 11, 13, and 14, 2020. They were challenging due to the low maximum elongation of this target (about 37 deg during our observations).

We obtained two visible spectra with the ACAM/WHT and with the ALFOSC/NOT instruments. The near-infrared part of the spectrum was obtained with the LIRIS/WHT instrument using the $I r-z j$ prism. It covers the 0.9-1.5 $\mu \mathrm{m}$ wavelength range. The merged spectrum is shown in Fig. 1. 


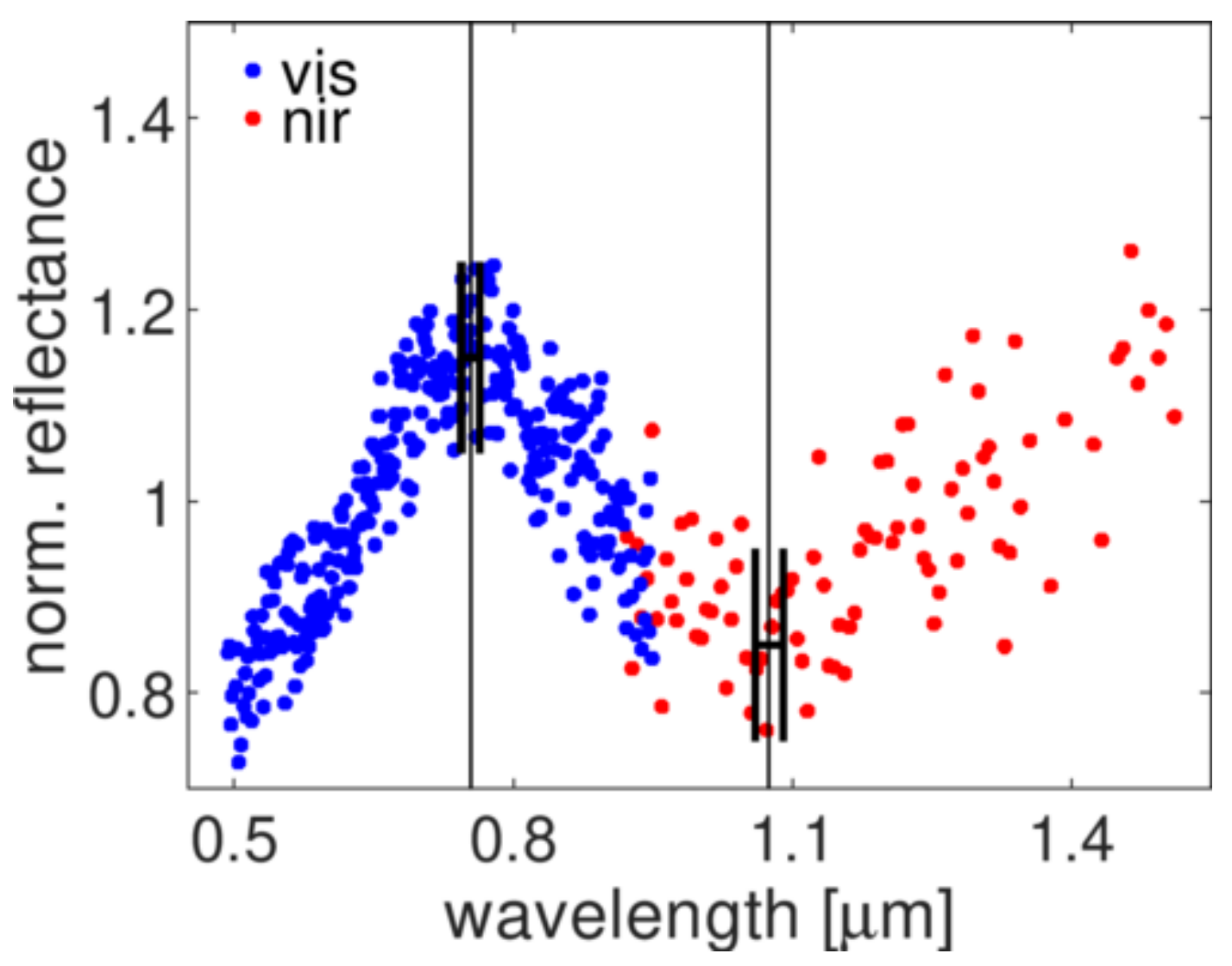

Fig. 1 The combined visible - near infrared spectrum of 2020 AV2 (Popescu et al. 2020). The spectral curve is normalized to unity at $1.25 \mu \mathrm{m}$. The reflectance maximum at $0.745 \mu \mathrm{m}$ and the band minimum at $1.075 \mu \mathrm{m}$ are outlined by the two markers.

The merged spectrum, covering a wavelength interval between 0.5 and $1.5 \mu \mathrm{m}$, allowed us to classify 2020 AV2 as an Sa-type asteroid. The value estimated for the $1 \mu \mathrm{m}$ band center, BIC $=1.08$ $\pm 0.020 \mu \mathrm{m}$, points towards a composition similar to that of the S(I) subtype of asteroids with olivine-pyroxene mixtures, defined by Gaffey et al. (1993). This value of BIC is indicative of a ferroan olivine mineralogy similar to that of brachinite meteorites.

Last but no least, we derived the effective diameter of this Vatira to be $1.50^{+1.10}-0.65 \mathrm{~km}$ by considering the average albedo of A-type and S-complex asteroids ( $\mathrm{pV}=0.23_{-0.08}{ }^{+0.11}$ ), and the absolute magnitude $(\mathrm{H}=16.40 \pm 0.78 \mathrm{mag})$.

References: [1] Bolin et al. 2020, MPEC 2020-A99; [2] de la Fuente Marcos \& de la Fuente Marcos, 2020, MNRAS, 494, L6; [3] Granvik et al. 2018, Icarus, 312, 181; [4] Greenstreet et al. 2012, Icarus, 217, 355; [5] Greenstreet 2020, MNRAS, 493, L129; [6] Popescu et al. 2020, MNRAS, paper accepted, https://doi.org/10.1093/mnras/staa1728

Acknowledgements: This work was developed in the framework of EURONEAR collaboration and of ESA P3NEOI projects. The work of M.P. was supported by a grant of the Romanian National Authority for Scientific Research - UEFISCDI, project number PN-III-P1-1.2-PCCDI-2017-0371. M.P., J.dL. and J.L. acknowledge support from the AYA2015-67772-R (MINECO, Spain), and from the European Union's Horizon 2020 research and innovation programme under grant agreement No 870403 (project NEOROCKS). This work was partially supported by the Spanish MINECO under grant ESP2017-87813-R. Based on observations made with the William Herschel Telescope (operated by the Isaac Newton Group of Telescopes) and the Nordic Optical Telescope. The paper make use of data published by the following web-sites Minor Planet Center, JPL Small-Body Database Browser , SMASS - Planetary Spectroscopy at MIT. 
